\title{
SOME NONZERO HOMOTOPY GROUPS OF SPHERES
}

\author{
BY EDWARD B. CURTIS
}

Communicated by William Browder, December 12, 1968

1. The purpose of this note is to establish some nonzero elements in the homotopy groups of spheres. This results from unstabilizing a method of Adams. Namely, an Adams spectral sequence is used to detect elements in $\pi_{n+i}\left(S^{n}\right)$ for various $n$ and $i$; in addition to the $d$ and $e$ invariants of Adams, the Hopf invariants are used to show that certain of these elements are nonzero. One consequence will be the following.

Consequence. The groups $\pi_{4+i}\left(S^{4}\right)$ are nonzero for all $i \geqq 0$.

2. Recall the mod-p-restricted lower central series spectral sequence (abbr: mod-p-RLCSSS), constructed as in [4], [5] and [10]. For each simplicial set $X$, form $G X$ as in [6], filter $G X$ by its mod- $p$ RLCS, and pass to the homotopy exact couple. The resulting spectral sequence we will label $E_{s, d}^{r}(X)$, where $s=$ filtration and $d=$ dimension. The results of $[4, \S(2.4)]$ show that for the sphere spectrum $S$, the term $E^{1}(S)$ of the mod-2-RLCSS is a ring $\Lambda$, with multiplicative generators $\lambda_{i}$ for each $i \geqq 0$. An additive basis for $E^{1}(S)$ consists of all monomials $\lambda_{I}=\lambda_{i_{1}} \cdots \lambda_{i_{k}}$, where $I=\left(i_{1}, \cdots, i_{k}\right)$ is a sequence of nonnegative integers with $2 i_{j} \geqq i_{j+1}$ for $j=1,2, \cdots, k-1$. Call such monomials allowable. In the unstable case, the results of $[4, \S(5.4)]$ show that for the $n$-sphere $S^{n}, E^{1}\left(S^{n}\right)$ is the subvector space of $\Lambda$ with basis all $\lambda_{I}$ which are allowable and for which $i_{1}<n$. Such a monomial $\lambda_{I} \in E^{1}\left(S^{n}\right)$, where $I=\left(i_{1}, \cdots, i_{k}\right)$, has filtration $k$, and dimension $n+\sum i_{j}$.

3. There is a short exact sequence of differential vector spaces:

$$
\left.0 \rightarrow E_{s, n+i}^{1}\left(S^{n}\right) \stackrel{i}{\rightarrow} E_{s, n+i+1}^{1}\left(S^{n+1}\right) \stackrel{h}{\rightarrow} E_{s-1, n+i+1}^{1} S^{2 n+1}\right) \rightarrow 0
$$

where $i$ is the inclusion and $h$ is defined on the allowable basis by

$$
\begin{aligned}
h\left(\lambda_{j} \lambda_{I}\right) & =\lambda_{I} & & \text { for } j=n, \\
& =0 & & \text { for } j<n .
\end{aligned}
$$

From this, there derives a long exact sequence

$$
\cdots \rightarrow E^{2}\left(S^{n}\right) \stackrel{i_{*}}{\rightarrow} E^{2}\left(S^{n+1}\right) \stackrel{h_{*}}{\rightarrow} E^{2}\left(S^{2 n+1}\right) \stackrel{\circ}{\rightarrow} \cdots
$$

It can be shown that $h_{*}$ commutes with all differentials, and is induced 
by the Hopf-invariant in the SHP-sequence of Whitehead, James:

$$
\cdots \rightarrow \pi_{n+1}\left(X^{n}\right) \stackrel{S}{\rightarrow} \pi_{n+i+1}\left(S^{n+1}\right) \stackrel{H}{\rightarrow} \pi_{n+i+1}\left(S^{2 n+1}\right) \stackrel{P}{\rightarrow} \cdots .
$$

From the sequence (3.1), some calculations in $E^{2}\left(S^{n}\right)$ can easily be made.

4. For each $m \geqq 0$, define functions $\phi_{2}(m), \phi_{3}(m), \phi_{4}(m), \phi_{5}(m), \phi(m)$ by the rules:

\begin{tabular}{|c|c|c|c|c|c|c|c|c|}
\hline$m=8 k+$ & 0 & 1 & 2 & 3 & 4 & 5 & 6 & 7 \\
\hline$\phi_{2}(m)=4 k+$ & 0 & 1 & 2 & 3 & 4 & 4 & 5 & 4 \\
$\phi_{3}(m)=4 k+$ & 0 & 1 & 2 & 3 & 3 & 4 & 3 & 4 \\
$\phi_{4}(m)=4 k+$ & 0 & 1 & 2 & 3 & 3 & 4 & 4 & 4 \\
$\phi_{5}(m)=4 k+$ & 0 & 1 & 2 & 3 & 3 & 4 & 4 & 4 \\
$\phi(m)=4 k+$ & 0 & 1 & 2 & 3 & 3 & 3 & 3 & 4 \\
\hline
\end{tabular}

The function $\phi(m)$ describes the Adams vanishing line: $\operatorname{Ext}_{A_{2}}^{s . l}\left(Z_{2}, Z_{2}\right)$ $=0$ for $s>\phi(t-s)$. Unstably, the functions $\phi_{n}(m)\left(\right.$ set $\phi_{n}(m)=\phi(m)$ for $n \geqq 6$ ) also describe a vanishing line, possibly modulo a tower, as follows.

TheOREm. $E_{s, n+i}^{2}\left(S^{n}\right)=0$ for $s>\phi_{n}(i)$, except for the tower at $i=0$, and the tower which occurs when $n$ is even and $i=n-1$.

This can be proven using the stable vanishing line $\phi(m)$ of Adams [1], (3.1), and downward induction.

COROLlARY. In the 2-component of $\pi_{n+i}\left(S^{n}\right)$, each element has order $\leqq 2^{\phi_{n}(i)}$.

This is of course the unstable analogue of $[1$, p. 69]. There is also a similar vanishing line for each prime $p$, and all together give a bound for the order of any element (of finite order).

5. Let $P$ be the periodicity operator defined by the Massey product $P(x)=\left\{x, \lambda_{0}^{4}, \lambda_{7}\right\}$. The following table describes some (not all) nonzero elements in $E^{2}\left(S^{n}\right)$ near the vanishing line. They are cycles in every $E^{r}\left(S^{n}\right)$ for which they are defined, as the differentials on them land in the vanishing-zone or in a tower. 
TABLE

\begin{tabular}{c|c|c|c|c}
\hline Stem dim $i$ & Filtration $s$ & $\begin{array}{c}\text { Minimum } \\
\text { value of } n\end{array}$ & Element in $E^{2}\left(S^{n}\right)$ & $\begin{array}{c}\text { Stable element } \\
\text { in Ext }\left(Z_{2}, Z_{2}\right)\end{array}$ \\
\hline $8 k$ & $4 k-1$ & 3 & $P^{k-1}\left(\lambda_{2} \lambda_{3}^{2}\right)$ & $P^{k-1}\left(c_{0}\right)$ \\
$8 k+1$ & $4 k$ & 2 & $P^{k-1}\left(\lambda_{1} \lambda_{2} \lambda_{3}^{2}\right)$ & $P^{k-1}\left(h_{1} c_{0}\right)$ \\
& $4 k+1$ & 3 & $P^{k}\left(\lambda_{1}\right)$ & $P^{k}\left(h_{1}\right)$ \\
$8 k+2$ & $4 k+2$ & 2 & $P^{k}\left(\lambda_{1}^{2}\right)$ & $P^{k}\left(h_{1}^{2}\right)$ \\
$8 k+3$ & $4 k+1$ & 5 & $P^{k}\left(\lambda_{3}\right)$ & $P^{k}\left(h_{2}\right)$ \\
& $4 k+2$ & 3 & $P^{k}\left(\lambda_{2} \lambda_{1}\right)$ & $P^{k}\left(h_{0} h_{2}\right)$ \\
& $4 k+3$ & 2 & $P^{k}\left(\lambda_{1}^{3}\right)$ & $P^{k}\left(h_{0}^{2} h_{2}\right)$ \\
$8 k+4$ & $4 k+2$ & 4 & $P^{k}\left(\lambda_{3} \lambda_{1}\right)$ & 0 \\
& $4 k+3$ & 3 & $P^{k}\left(\lambda_{2} \lambda_{1}^{2}\right)$ & 0 \\
$8 k+5$ & $4 k+3$ & 4 & $P^{k}\left(\lambda_{3} \lambda_{1}^{2}\right)$ & 0 \\
$8 k+6$ & $4 k+4$ & 3 & $P^{k}\left(\lambda_{2} \lambda_{1}^{3}\right)$ & 0 \\
$8 k+7$ & $4 k+4$ & 4 & $P^{k}\left(\lambda_{3} \lambda_{1}^{3}\right)$ & 0 \\
& $4 k+4$ & 5 & $P^{k}\left(\lambda_{7} \lambda_{0}^{3}\right)$ & $P^{k}\left(h_{0}^{3} h_{3}\right)$ \\
\hline
\end{tabular}

The elements $P^{k-1}\left(c_{0}\right), P^{k-1}\left(h_{1} c_{0}\right), P^{k}\left(h_{7}\right), P^{k}\left(h_{1}^{2}\right), P^{k}\left(h_{2}\right), P^{k}\left(h_{0} h_{2}\right)$, $P^{k}\left(h_{0}^{2} h_{2}\right), P^{k}\left(h_{0}^{3} h_{3}\right)$ are shown never to be boundaries in the stable Adams spectral sequence because of nonzero $d$ or $e$ invariants; see [2], [7], [8], [9]. Hence, by naturality of suspension, their precursors are never boundaries in each $E^{r}\left(S^{n}\right)$ of the mod-2-RLCSSS.

The Hopf-invariant $h_{*}: E^{r}\left(S^{3}\right) \rightarrow E^{r}\left(S^{5}\right)$ shows that the elements $P^{k}\left(\lambda_{2} \lambda_{1}^{2}\right), P^{k}\left(\lambda_{2} \lambda_{1}^{3}\right)$ are not boundaries in any $E^{r}\left(S^{3}\right)$, since $h_{*}$ of them are not boundaries in $E^{r}\left(S^{5}\right)$. Similarly, the elements $P^{k}\left(\lambda_{3} \lambda_{1}\right), P^{k}\left(\lambda_{3} \lambda_{1}^{2}\right)$ and $P^{k}\left(\lambda_{3} \lambda_{1}^{3}\right)$ are never boundaries in any $E^{r}\left(S^{4}\right)$.

6. For odd primes $p$, the $E^{1}$-term of the mod-p-RLCSSS for odd spheres is described in $[4, \S 8]$. The analogous vanishing statement is $E_{s, n+i}^{2}\left(S^{n}\right)=0$, for all odd $n$, and $s>[i+3 / 2 p-2]$. Also, in filtration $k$ and dimension $3+2 k(p-1)-1, E^{2}\left(S^{3}\right)$ has a single generator say $a_{k}$. As all differentials on $a_{k}$ land in the vanishing zone, $a_{k}$ is a permanent cycle; also, $a_{k}$ is never a boundary, shown by a mod- $p$ version of [9]. Thus $a_{k}$ detects a nonzero class of order $p$ in $\pi_{3+2 k(p-1)-1}\left(S^{3}\right)$. Of course the element detected by $a_{k}$ is just (a nonzero multiple of) Toda's $\alpha_{k}$ shown to be nonzero by Adams' $e$-invariant argument.

7. It is now easy to exhibit some nonzero homotopy classes, as each of the elements in the table detects a nonzero class in $\pi_{*}\left(S^{n}\right)$ for the corresponding value of $n$. Using also the elements $\alpha_{k}(3)$ for stems 
$\equiv 7(\bmod 8)$, there follows consequence $(1)$. Further, $\pi_{3+i}\left(S^{3}\right)$ is nonzero at least for all $i \neq \equiv 6(\bmod 8)$, and hence also $\pi_{2+i}\left(S^{2}\right)$ is nonzero at least for all $i \neq 7(\bmod 8)$.

\section{BIBLIOGRAPHY}

1. J. F. Adams, Stable homotopy theory, Springer-Verlag, Berlin, 1964.

2. - On the groups $J(X)$. IV, Topology 5 (1966), 21-71.

3. M. G. Barratt (unpublished).

4. A. K. Bousfield et al., The mod-p-lower central series and the Adams spectral sequence, Topology 5 (1966), 331-342.

5. E. B. Curtis, Simplicial homotopy theory, Lecture Notes, Aarhus University, Denmark, 1967.

6. D. M. Kan, A combinatorial definition of homotopy groups, Ann. of Math (2) 67 (1958), 282-312.

7. M. Mahowald, The meta-stable homotopy of $S^{n}$, Mem. Amer. Math. Soc. No. 72, 1967.

8. - On the order of the image of $J$, Topology 6 (1967), 371-378.

9. C. R. F. Maunder, On the differentials in the Adams spectral sequence for the stable homotopy groups of spheres. I, II, Proc. Cambridge Philos. Soc. 61 (1965), 53-60, 855-868.

10. D. L. Rector, An unstable Adams spectral sequence, Topology 5 (1966), 343-396.

11. H. Toda, Composition methods in homotopy groups of spheres, Ann. of Math. Studies No. 49, Princeton Univ. Press, Princeton, N. J., 1962.

Massachusetts Institute of Technology,

Cambridge, Massachusetts 02139 